\title{
Syndrome of pleural and retrosternal "bridging" fibrosis and retroperitoneal fibrosis in patients with asbestos exposure
}

\author{
V Cottin, ${ }^{1}$ P-Y Brillet, ${ }^{2} \mathrm{~F}$ Combarnous, ${ }^{3} \mathrm{~F}$ Duperron, ${ }^{2} \mathrm{H}$ Nunes, ${ }^{4} \mathrm{~J}-\mathrm{F}$ Cordier ${ }^{1}$
}

${ }^{1}$ Hospices Civils de Lyon, Lyon I University, Louis Pradel Hospital, Department of Respiratory Diseases - Reference Center for Orphan Lung Disorders, Lyon, France; ${ }^{2}$ Assistance Publique Hôpitaux de Paris, Paris 13 University, Avicenne Hospital, Department of Imaging, Bobigny, France; ${ }^{3}$ Clinique du Tonkin, Department of Nephrology, Villeurbanne, France; ${ }^{4}$ Assistance Publique Hôpitaux de Paris, Paris 13 University, Avicenne Hospital, Department of Respiratory Diseases, Bobigny, France

Correspondence to: Pr V Cottin, Hôpital Louis Pradel, 28 avenue Lépine, 69677 Lyon (Bron) Cedex, France; vincent. cottin@chu-lyon.fr

Received 22 March 2007 Accepted 27 May 2007

\section{ABSTRACT}

Two case histories are described of pleural and anterior mediastinal fibrosis presenting as a continuous fibrotic process with thick parietal pleural plaques extending from one pleura to the contralateral pleura through the retrosternal area, and with retroperitoneal fibrosis. Followup over 4 years in one case demonstrated rapid progression of disease, with pleural fibrosis preceding retrosternal and retroperitoneal fibrosis. Histopathological analysis in both cases showed non-tumoral fibrosis with broad fibrous bundles surrounding fibroblasts (and lymphocytes in one case). Possible causes such as infections and exposure to ergot derivatives were excluded. Both patients had been slightly or moderately exposed to asbestos. These cases represent an unusual new presentation of pleural and retrosternal fibrosis extending beyond the anatomical structures and associated with retroperitoneal fibrosis.

We report a syndrome of pleural and retrosternal "bridging" fibrosis presenting as a single continuous fibrotic process associated with retroperitoneal fibrosis and probably related to asbestos exposure.

\section{CASE REPORTS}

\section{Case 1}

A 65-year-old man was admitted with acute renal failure. He was a smoker of 50 pack-years. Thirty years earlier he had been exposed to asbestos without respiratory protection for 5 years. He denied taking any medication other than amlodipine. Physical examination was normal except for slight supraclavicular oedema. Serum creatinine was $500 \mu \mathrm{mol} / \mathrm{l}$. Renal ultrasonography showed stasis and caliceal dilation of the left kidney and a small right kidney. Abdominal CT and MRI suggested retroperitoneal fibrosis with a hypodense soft tissue retroperitoneal mass, $40 \mathrm{~mm}$ in diameter, extending from the left kidney to the aorta. The CT scan showed bilateral ureteral stenosis, a non-functional atrophic right kidney and thrombosis of the splenic and left renal veins. An exploratory laparotomy showed a hard fibrous mass in the retroperitoneum adherent to adjacent vessels. Renal function improved following endoureteral catheterisation.

The chest radiograph showed reduced lung volumes with opacities of the right apex and the retrosternal area (fig 1A, B). The patient had mild dyspnoea on exertion. The CT scan showed several calcified parietal pleural plaques and a thick calcified mass that extended ("bridged") from one pleura to the contralateral pleura, with mediastinal retrosternal involvement (fig $1 \mathrm{C}$ ). Positron emis- sion tomography showed no increased uptake of fluorodeoxyglucose. Pulmonary function tests showed forced vital capacity (FVC) 79\% of predicted, total lung capacity (TLC) 67\%, forced expiratory volume in $1 \mathrm{~s}\left(\mathrm{FEV}_{1}\right) 85 \%$ predicted, $\mathrm{FEV}_{1} / \mathrm{FVC} 82 \%$. Carbon monoxide transfer coefficient (KCO) was 101\% predicted. Arterial blood gases were normal. Differential measurements of immunoglobulins (Ig) were normal, including $\mathrm{IgG}_{4}$ levels.

Histopathological examination of large surgical biopsy specimens of the retroperitoneal mass showed severe fibrosis with broad fibrous strands and a few fibroblasts. Immunohistological analysis demonstrated positive staining with anti-smooth muscle actin antibody, and negative staining using anti-CD34, anti-MDM2 and anti-CDK4 antibodies. A specimen obtained by trans-sternal biopsy of the retrosternal mass ( $15 \mathrm{~mm}$ thick) was very similar to the retroperitoneal tissue.

The patient was treated with oral prednisone for 6 months $(1 \mathrm{mg} / \mathrm{kg} /$ day for 1 month then progressively tapered) with no change in the chest CT scan and pulmonary function. Tamoxifen was started (40 mg/day) and, after 4 months of treatment, FVC and TLC had both increased by 0.3 litres and the chest CT scan was unchanged.

\section{Case 2}

A 55-year-old man was admitted in 2001 with chronic thoracic pain, fatigue and moderate dyspnoea on exertion. He had smoked 50 pack-years. He denied taking any medication. He had been exposed to lead and trichlorethylene as a print worker and had worked in a building with asbestos-flocked walls for 4 years. The chest CT scan showed bilateral calcified circumferential pleural plaques predominating on the anterior parietal pleura and a moderate left pleural effusion (fig 2A). Diagnostic thoracentesis showed a protein level of $55 \mathrm{~g} / \mathrm{l}$ with $50 \%$ lymphocytes, $24 \%$ neutrophils, $15 \%$ mesothelial cells and $11 \%$ eosinophils. FVC was $60 \%$ predicted, TLC $60 \%$ predicted, $\mathrm{FEV}_{1} 54 \%$ predicted, $\mathrm{FEV}_{1} / \mathrm{FVC} 70 \%$ and Kco $126 \%$ predicted. Serum protein electrophoresis showed a moderate IgG kappa oligoclonal fraction with increased total IgG, normal IgA and reduced IgM levels. A myelogram showed 5\% normal plasmocytes.

Left videothoracoscopic surgery showed diffuse pleural thickening involving both the parietal and visceral pleura. Histopathological examination of large parietal pleural biopsy specimens showed severe fibrosis with large bundles of collagen surrounding infiltrates rich in lymphocytes, fibroblasts, some plasma cells and a few histiocytes 

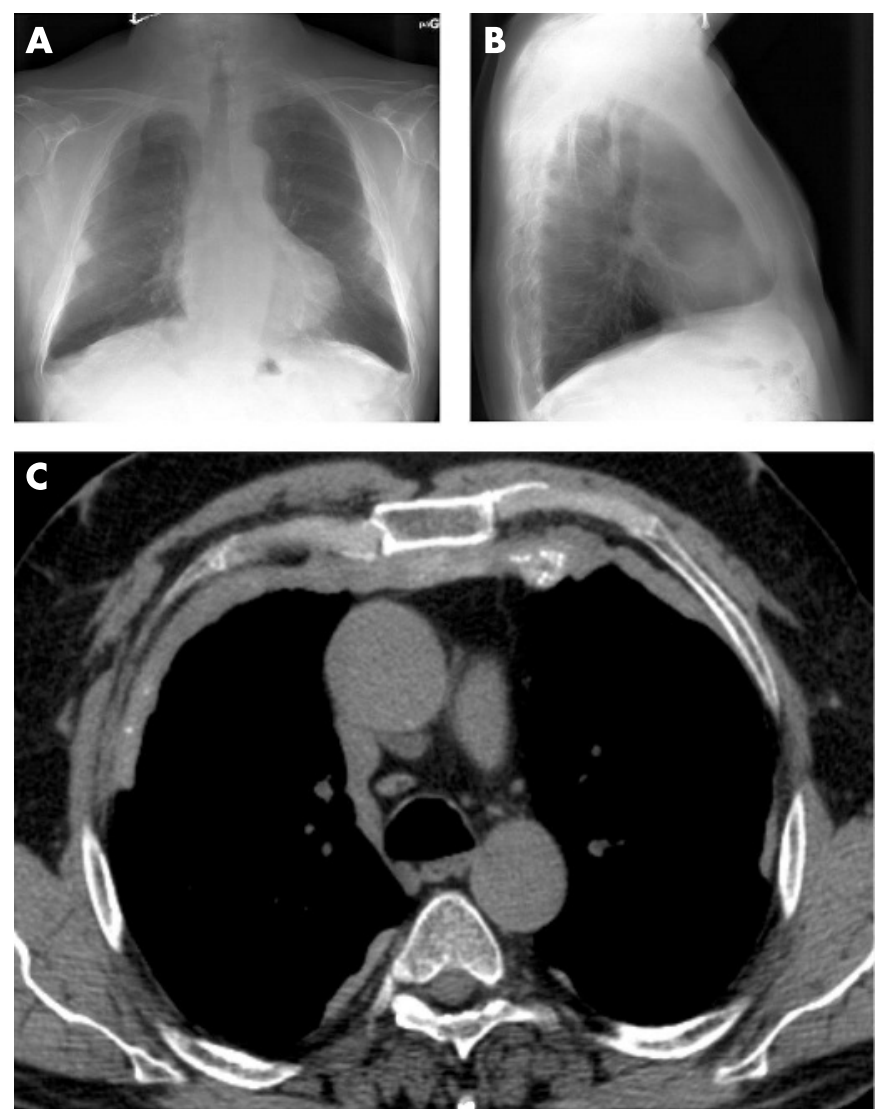

Figure 1 Retrosternal and pleural fibrosis in patient 1. (A) Anterior posterior chest radiograph showing reduced lung volumes, pleural opacity of the right lung apex and axillary pleural plaques. (B) Lateral view of the chest radiograph showing enlargement of the retrosternal area. (C) CT scan of the chest showing a dense fibrous lesion extending from one pleura to the contralateral pleura through the retrosternal area ("bridging fibrosis").

with a palisade distribution. No granuloma, vasculitis or tumour was found. Immunocytological analysis of the lymphocytes excluded tumoral proliferation. Because of thoracic pain, the patient was treated with oral prednisone $(30 \mathrm{mg} /$ day) for 18 months with improvement in symptoms and resolution of the pleural effusion.

In 2005 he was readmitted with thoracic pain and weight loss $(10 \mathrm{~kg})$. The chest CT scan showed considerable worsening of the pleural plaques with left circumferential pleural thickening, right anterolateral and posterior pleural plaques, and a thick calcified retrosternal mass "bridging" from one pleura to the other through the anterior mediastinum (fig 2B). An abdominal CT scan showed thick hypodense soft tissue around the aorta extending to the primitive iliac arteries, suggesting retroperitoneal fibrosis (fig 2C). Positron emission tomography showed a slightly increased uptake of fluorodeoxyglucose in the pleural plaques which did not indicate malignancy. Pulmonary function tests, serum and urine protein electrophoresis and serum Ig were unchanged. The serum $\operatorname{IgG}_{4}$ level was normal. In October 2006 he was alive with no evidence of myeloma on the myelogram.

\section{DISCUSSION}

Pleural, mediastinal and retroperitoneal fibrosis usually present as distinct entities. Pleural fibrosis is most commonly caused by asbestos exposure and may follow asbestos-related benign pleural effusion, ${ }^{1}$ tuberculous pleural effusion, haemothorax,
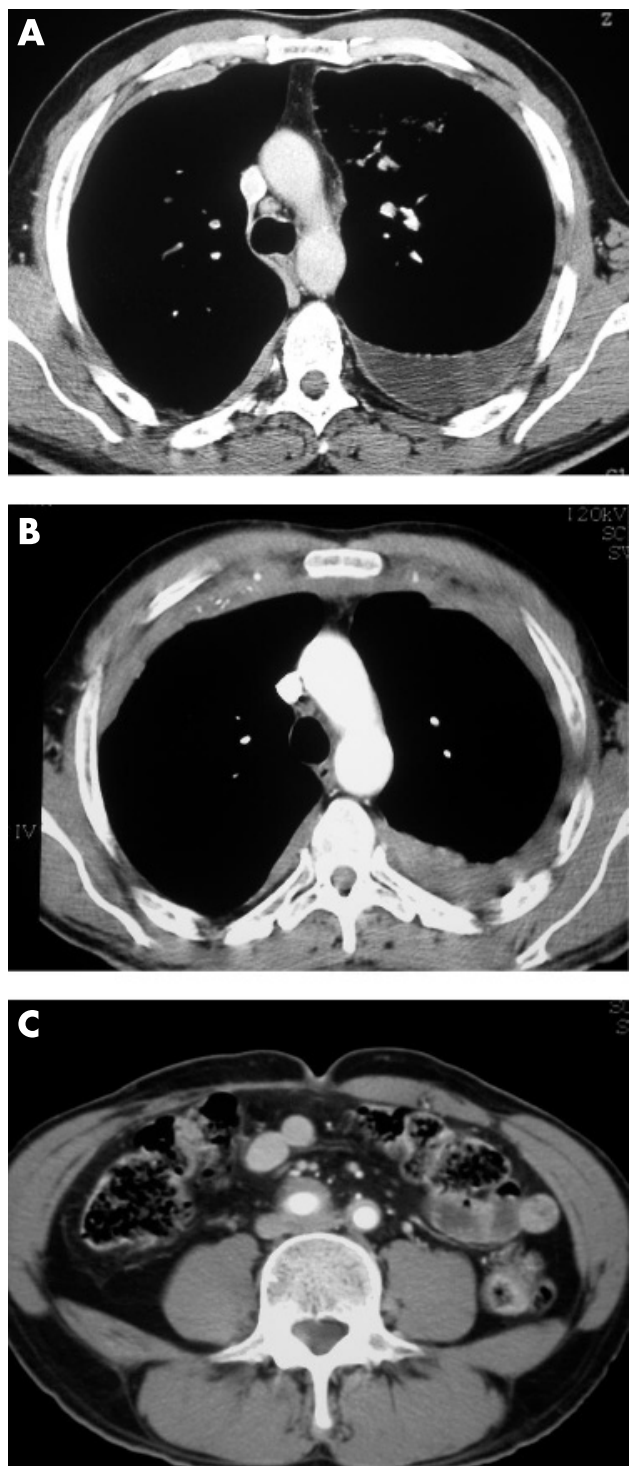

Figure 2 Retrosternal, pleural and retroperitoneal fibrosis in patient 2. (A) CT scan of the chest in 2001 showing a moderate left pleural effusion and bilateral pleural thickening predominating on the anterior parietal pleura. (B) CT scan of the chest in 2005 showing dramatic worsening of the bilateral pleural thickening with left circumferential pleural thickening and a thick calcified retrosternal mass in continuity with the pleura on both sides ("bridging fibrosis"). (C) CT scan of the abdomen in 2005 showing periaortic retroperitoneal fibrosis.

use of ergot derivatives, and occasionally rheumatoid pleurisy, uremic pleuritis or coronary artery bypass graft surgery; rarely it may remain idiopathic, ${ }^{12}$ occasionally in a familial context. ${ }^{34}$ Mediastinal fibrosis may develop as a consequence of histoplasmosis or tuberculosis, or rarely actinomycosis, syphilis, aspergillosis, sarcoidosis, silicosis or methysergide exposure, or it may remain idiopathic. ${ }^{5}$ Retroperitoneal fibrosis is generally idiopathic and may occasionally be secondary to the use of drugs, asbestos exposure, malignant disease, infections, radiotherapy or surgery. ${ }^{6}$

The imaging presentation of pleural fibrosis is quite distinct from that of mediastinal fibrosis. Asbestos-related pleural fibrosis may present as parietal pleural plaques, often bilateral and partially calcified, or less frequently as diffuse visceral pleural thickening. ${ }^{1}$ It may be associated with apical lung fibrosis $^{3}$ or be asymmetrical. ${ }^{2}{ }^{7}$ Conversely, three presentations 
of mediastinal fibrosis have been suggested: ${ }^{5}$ granulomatous mediastinitis (often resulting from tuberculosis) with enlarged mediastinal lymph nodes and usually asymptomatic; mediastinal fibrosis associated with histoplasmosis characterised by a focal, invasive and calcified process; and diffuse proliferation of non-calcified fibrous tissue causing major encasement of normal structures (typical of idiopathic mediastinal fibrosis). Retrosternal fibrosis with direct continuity with pleural fibrosis (itself not anatomically connected to retroperitoneal fibrosis) as observed in both our patients is clearly distinct from these previous descriptions.

Despite some overlap of potential causes, pleural and mediastinal fibrosis have not previously been considered as associated manifestations. Mediastinal and retroperitoneal fibrosis may occasionally be associated. ${ }^{8-10}$ Concomitant pleural and retroperitoneal fibrosis have been reported in patients exposed to asbestos. ${ }^{11}{ }^{12}$ Fibrosis of several organs (multifocal fibrosclerosis) has been reported with involvement of almost any organ. ${ }^{13}$ Fibrosis may also involve the lung parenchyma adjacent to pleural fibrosis. ${ }^{3}$ Nevertheless, a recent report on retroperitoneal fibrosis related to asbestos exposure ${ }^{14}$ included chest CT images which were strikingly similar to our patients (the authors did not comment on the unusual imaging appearance).

Both our patients had some exposure to asbestos, although it was mild to moderate and short term. Since it is well known that asbestos induces pleural and retroperitoneal fibrosis, ${ }^{14} 15$ exposure to asbestos may be considered as a causative agent in this unusual presentation. All other potential causes were carefully ruled out, including elevation of $\mathrm{IgG}_{4}$ involved in idiopathic mediastinal and retroperitoneal fibrosis. ${ }^{9}$ In case 2 pleural fibrosis was preceded by mediastinal and retroperitoneal fibrosis for several years, which argues against the possibility of an infectious process such as tuberculosis or histoplasmosis (Histoplasma capsulatum is not endemic in France).

Disease progression was more rapid than is usually seen in patients with asbestos-related pleural fibrosis. No improvement was observed with corticosteroid treatment. Tamoxifen has been shown to be beneficial in isolated cases of retroperitoneal fibrosis. ${ }^{16}$

Acknowledgements: The authors thank Dr D Ranchère-Vince and Professor $F$ Thivolet-Béjui (Lyon) for pathological analysis; and Professor J P Battesti (Bobigny), Dr B Etienne-Mastroïanni and Dr Pierre Faurie (Lyon) for the clinical care of the patients.

Funding: None

Competing interests: None.

\section{REFERENCES}

1. Jantz MA, Antony VB. Pleural fibrosis. Clin Chest Med 2006;27:181-91.

2. Buchanan DR, Johnston ID, Kerr IH, et al. Cryptogenic bilateral fibrosing pleuritis. Br J Dis Chest 1988;82:186-93.

3. Azoulay E, Paugam B, Heymann MF, et al. Familial extensive idiopathic bilateral pleural fibrosis. Eur Respir J 1999;14:971-3.

4. Hayes JP, Wiggins J, Ward K, et al. Familial cryptogenic fibrosing pleuritis with Fanconi's syndrome (renal tubular acidosis). A new syndrome. Chest 1995;107:576-

5. Parish JM, Rosenow EC 3rd. Mediastinal granuloma and mediastinal fibrosis. Semin Respir Crit Care Med 2002;23:135-43.

6. Vaglio A, Salvarani C, Buzio C. Retroperitoneal fibrosis. Lancet 2006;367:241-51.

7. Lee-Chiong TL Jr, Hilbert J. Extensive idiopathic benign bilateral asynchronous pleural fibrosis. Chest 1996;109:564-5.

8. Szarf G, Bluemke DA. Case 83: Multifocal fibrosclerosis with mediastinalretroperitoneal involvement. Radiology 2005:235:829-32.

9. Zen Y, Sawazaki A, Miyayama S, et al. A case of retroperitoneal and mediastinal fibrosis exhibiting elevated levels of lgG4 in the absence of sclerosing pancreatitis (autoimmune pancreatitis). Hum Pathol 2006;37:239-43.

10. Morad N, Strongwater SL, Eypper S, et al. Idiopathic retroperitoneal and mediastinal fibrosis mimicking connective tissue disease. Am J Med 1987;82:363-6.

11. Maguire GP, Meggs LG, Addonizio J, et al. Association of asbestos exposure, retroperitoneal fibrosis, and acute renal failure. NY State J Med 1991:91:357-9.

12. Boulard JC, Hanslik T, Doleris LM, et al. Asbestos and idiopathic retroperitoneal fibrosis. Lancet 1995;345:1379.

13. Leung WS, Yuen NW, Chan VL, et al. A 55-year-old man with fever together with submandibular and lung masses. Chest 2006;129:488-92.

14. Uibu T, Oksa $P$, Auvinen $A$, et al. Asbestos exposure as a risk factor for retroperitoneal fibrosis. Lancet 2004;363:1422-6.

15. Hillerdal G. Pleural plaques in a health survey material. Frequency, development and exposure to asbestos. Scand J Respir Dis 1978;59:257-63.

16. Owens LV, Cance WG, Huth JF. Retroperitoneal fibrosis treated with tamoxifen. Am Surg 1995;61:842-4. 